\title{
A Comprehensive Attribute Assessment Model for Evaluating Teaching Quality of University
}

\author{
Wenyu Li1 \\ School of Mathematics and Statistics \\ Beihua University \\ Jilin China \\ liwenyu82@163.com
}

\author{
Yunlong Lu \\ School of Mathematics and Statistics \\ Beihua University \\ Jilin China \\ luyunlong78@163.com
}

\begin{abstract}
A comprehensive attribute assessment model is proposed for evaluating teaching quality of all teaching units in a university. By establishing the Index System of undergraduate teaching level of Ordinary colleges and Universities in China and using the attribute assessment system and the objective weight, the teaching quality of all teaching units is evaluated. The ranking and the evaluation grade for units are presented. It shows that the model is feasible and reliable for evaluating teaching quality. Furthermore, the assessment presented could give references for related teaching administers.
\end{abstract}

Keywords-teaching quality evaluation; index system; comprehensive attribute assessment; objective weight

\section{INTRODUCTION}

In recent years, higher education enrollment scale in China continues to be expanded. The higher education infrastructures for teaching, the quality and quantity of teaching staff are highlighted. It is a potential threat to the quality of teaching in colleges and universities. Hence, it is very important to carry out the research on the evaluation of teaching quality in Colleges and universities. At present, the major methods for the teaching quality evaluation are Analytical Hierarchy Process (AHP) method, the mathematical statistics method, the artificial neural network method, ant the fuzzy comprehensive evaluation method [1-4]. The attribute comprehensive assessment method can solve effectively the ordered partition identification problem, and have a good evaluation result [5]. Therefore, in this paper, we present a comprehensive attribute assessment model for teaching quality evaluation. The evaluation result provides some useful information and reference for teaching administers.

\section{AN ATTRIBUTE ASSESSMENT MODEL WITH OBJECTIVE WEIGHT}

Considering the space of research object $X, n$ samples $x_{1}, x_{2}, \cdots, x_{n}$, are chosen from $X . m$ Indexes for every sample are measured, denoted by $I_{1}, I_{2}, I_{3}, \cdots, I_{m} . x_{i j}$ denotes the ith index measure data of the jth sample, where $i=1,2, \cdots, m, j=1,2, \cdots, n$. Hence, the $\mathrm{jth}$

This work was supported by Jilin Province educational science"12th FiveYear"project(GH150078).

Corresponding author: Yunlong Lu. sample can be denoted by a vector $x_{j}=\left(x_{1 j}, x_{2 j}, \cdots, x_{m j}\right), j=1,2, \cdots, n$.

Let $F$ be an attribute space on $X,\left(C_{1}, C_{2}, \cdots, C_{K}\right)$ is an ordered partition on $F$, such that $C_{1}>C_{2}>\cdots>C_{K}$. The following table is the grading partition table.

TABLE I. THE GRADE PARTITION TABLE OF INDEXES

\begin{tabular}{|c|c|c|c|c|}
\hline & $C_{1}$ & $C_{2}$ & .......... & $C_{K}$ \\
\hline$I_{1}$ & $a_{10} \sim a_{11}$ & $a_{11} \sim a_{12}$ & ......... & $a_{1 K-1} \sim a_{1 K}$ \\
\hline .......... & .......... & ......... & ......... & ......... \\
\hline$I_{m}$ & $a_{m 0} \sim a_{m 1}$ & $a_{m 1} \sim a_{m 2}$ & $\ldots \ldots \ldots$ & $a_{m K-1} \sim a_{m K}$ \\
\hline
\end{tabular}

where $a_{i 0}<a_{i 1}<a_{i 2}<\cdots<a_{i K}$, or $a_{i 0}>a_{i 1}>a_{i 2}>\cdots>a_{i K}$.

\section{A. Single-index Attribute Measure}

The attribute measure $\mu_{i j k}=\mu_{i j k}\left(x_{i j} \in C_{k}\right)$ is computed, which denotes the attribute measure value of the ith index of the jth sample with the grade $C_{k}, k=1,2, \cdots, K$.

Let's suppose $a_{i 0}>a_{i 1}>a_{i 2}>\cdots>a_{i K}$. We set

$$
b_{i k}=\frac{a_{i k-1}+a_{i k}}{2}, k=1,2,3, \cdots K,
$$

and define

$$
d_{i k}=\min \left(\left|b_{i k}-a_{i k}\right|,\left|b_{i k+1}-a_{i k}\right|\right), k=1,2,3, \cdots, K-1 .
$$

The attribute measure value $\mu_{i j k}$ can be computed by

$$
\mu_{i j 1}= \begin{cases}1, & a_{i 1}+d_{i 1}<x_{i j}, \\ \frac{\left|x_{i j}-a_{i 1}+d_{i 1}\right|}{2 d_{i 1}}, & a_{i 1}-d_{i 1} \leq x_{i j} \leq a_{i 1}+d_{i 1}, \\ 0, & x_{i j}<a_{i 1}-d_{i 1},\end{cases}
$$




$$
\begin{aligned}
& \mu_{i j k}= \begin{cases}0, & a_{i k-1}+d_{i k-1}<x_{i j}, \\
\frac{\left|x_{i j}-a_{i k-1}-d_{i k-1}\right|}{2 d_{i k-1}}, & a_{i k-1}-d_{i k-1} \leq x_{i j} \leq a_{i k-1}+d_{i k-1}, \\
1, & a_{i k}+d_{i k} \leq x_{i j} \leq a_{i k-1}-d_{i k-1}, \\
\frac{\left|x_{i j}-a_{i k}+d_{i k}\right|}{2 d_{i k}}, & a_{i k}-d_{i k} \leq x_{i j} \leq a_{i k}+d_{i k}, \\
0, & x_{i j} \leq a_{i k}-d_{i k},\end{cases} \\
& \mu_{i j K}= \begin{cases}\frac{1,}{\left|x_{i j}-a_{i K-1}-d_{i K-1}\right|}, & a_{i K-1}-d_{i K-1}, \\
2 d_{i K-1} & a_{i K-1}-d_{i K-1}<x_{i j} \leq a_{i K-1}+d_{i K-1}, \\
0, & \end{cases}
\end{aligned}
$$

From the above single-index attribute measure formula, we can compute the every index attribute measure value of the $j$ th sample. Next, we need compute the $j$ th sample $x_{j}$ attribute measure value $\mu_{j k}=\mu\left(x_{j} \in C_{k}\right)$. Because the important degree of each index is different, we need consider the weight of each index.

\section{B. Computing Weight}

In this paper, the index weights are computed by the entropy weight method. The entropy weight method is a class objective weight method. It can escape the expert's subjective preference. The ith index entropy weight is given by the following formula

$$
\omega_{i}=\frac{1-E_{i}}{m-\sum_{i=1}^{m} E_{i}}
$$

where $E_{i}=q \sum_{j=1}^{n} p_{i j} \ln p_{i j}, p_{i j}=\frac{x_{i j}}{\sum_{j=1}^{n} x_{i j}}, q=\ln n$, and if $p_{i j}=0$, then $p_{i j} \ln p_{i j}=0$.

\section{Multi-index Attribute Measure}

By using (1) and (2), the $j$ th sample $x_{j}$ multi-index attribute measure $\mu_{j k}=\mu\left(x_{j} \in C_{k}\right)$ can be computed by the formula

$$
\mu_{j k}=\mu\left(x_{j} \in C_{k}\right)=\sum_{i=1}^{m} \omega_{i} \mu_{i j k}, 1 \leq i \leq m, 1 \leq j \leq n .
$$

By the multi-index attribute measure value, we can compute the evaluation grade and ranking of each sample.

\section{Scoring criteria and Confidence Criterion}

Let $\left(C_{1}, C_{2}, \cdots, C_{K}\right)$ is an ordered partition on $F$, such that $C_{1}>C_{2}>\cdots>C_{K} \cdot n_{k}$ denotes the score of $C_{k}$. The attribute score of $x_{j}$ is defined as follow

$$
q_{x}=\sum_{k=1}^{K} n_{k} \mu\left(x_{j} \in C_{k}\right),
$$

where $n_{k}=\frac{\sum_{i=1} \frac{a_{i k-1}+a_{i k}}{2}}{m}, k=1,2, \cdots, K$.

Scoring criteria: If $q_{x}>q_{y}$, then $x$ is better than $y$.

Confidence criterion: Let $\lambda$ is a confidence criterion $(0.5 \leq \lambda<1)$. Compute

$$
k_{0}=\min \left\{k: \sum_{l=1}^{k} \mu\left(x_{j} \in c_{l}\right) \geq \lambda, 1 \leq k \leq K\right\}
$$

then $x_{j}$ belongs to the grade $C_{k_{0}}$.

\section{APPLICATION}

In this section, we will evaluate the teaching quality of 19 teaching units in a university. The evaluation indicator system of teaching quality on all teaching units is founded in [6], in which there are 35 indexes, among them 5 first grade indexes, 15 second grade indexes and 31third grade indexes, and 4 second grade indexes in second grade indexes have not third grade indexes. The grade partition table of indexes table and original measure data are also seen in [6]. Consider all teaching units, the attribute space

$$
F=\{\text { teaching quality }\} \text {. }
$$

Set

$$
\begin{aligned}
& C_{1}=\{\text { high satisfaction }\}, \\
& C_{2}=\{\text { satisfaction }\}, \\
& C_{3}=\{\text { basically satisfaction }\},
\end{aligned}
$$

then $\left\{C_{1}, C_{2}, C_{3}\right\}$ is an ordered partition of $F$, and

$$
C_{1}>C_{2}>C_{3}, K=3
$$

Next we present the realization concrete process of model.

Step 1, by the measure data for all teaching data, (1), (6) and the ordered partition table, the single-index attribute measure $\mu_{i j k}$ can be computed.

Step 2, by the measure data for all teaching data and (2), the entropy weight can be obtained.

Step 3, by (3) and the results from the above, the multi-index attribute measure value $\mu_{j k}=\mu\left(x_{j} \in C_{k}\right)$ can be obtained. Using (4) and (5), we can compute the attribute score and identify the evaluation grade.

From the second step, the entropy weights of indexes are computed, see Table II. 
TABLE II. THE WEIGHTS OF INDEXSES

\begin{tabular}{cccccccc}
\hline index & 1 & 2 & 3 & 4 & 5 & 6 & 7 \\
\hline weigh & 2.09 & 4.33 & 5.94 & 2.06 & 0.96 & 1.32 & 3.75 \\
\hline index & 8 & 9 & 10 & 11 & 12 & 13 & 14 \\
\hline weigh & 1.26 & 0.78 & 4.16 & 12.7 & 6.40 & 2.72 & 6.34 \\
\hline index & 15 & 16 & 17 & 18 & 19 & 20 & 21 \\
\hline weigh & 4.91 & 4.41 & 4.02 & 2.05 & 2.74 & 1.21 & 1.33 \\
\hline index & 22 & 23 & 24 & 25 & 26 & 27 & 28 \\
\hline weigh & 2.11 & 1.57 & 0.90 & 1.95 & 2.53 & 1.42 & 1.02 \\
\hline index & 29 & 30 & 31 & 32 & 33 & 34 & 35 \\
\hline weigh & 1.38 & 1.53 & 4.32 & 1.80 & 2.29 & 0.78 & 1.50
\end{tabular}

Next, we take the teaching unit HT for example to illustrate the above procedure. By Step 1, we compute the single-index attribute measure (see Table III). By Step 2, the multi-index attribute measure of the teaching unit HT is $\left(\mu_{11}, \mu_{12}, \mu_{13}\right)=(0.32,0.12,0.56)$, the attribute score $q_{\mathbf{H T}}=2.53$. As $0.32+0.12=0.44<0.5$, and $0.32+0.12+0.56=1>0.5$, hence the evaluation grade is basically satisfaction. In Table III, "I" denotes index, "MM" denotes multi-index attribute measure.

TABLE III. THE WEIGHTS OF INDEXSES

\begin{tabular}{llllll}
\hline I & MM & I & MM & I & MM \\
\hline 1 & $(0,0,1)$ & 13 & $(0,0,1)$ & 25 & $(0,0,1)$ \\
\hline 2 & $(0,0.38,0.62)$ & 14 & $(0,0,1)$ & 26 & $(0,0,1)$ \\
\hline 3 & $(0.67,0.33,0)$ & 15 & $(1,0,0)$ & 27 & $(0,0,1)$ \\
\hline 4 & $(0,0,1)$ & 16 & $(1,0,0)$ & 28 & $(0,0.27,0.73)$ \\
\hline 5 & $(1,0,0)$ & 17 & $(1,0,0)$ & 29 & $(0,0,1)$ \\
\hline 6 & $(1,0,0)$ & 18 & $(0,0,1)$ & 30 & $(0,0,1)$ \\
\hline 7 & $(0.24,0.76,0)$ & 19 & $(0,0,1)$ & 31 & $(1,0,0)$ \\
\hline 8 & $(1,0,0)$ & 20 & $(0,0,1)$ & 32 & $(0.91,0,0.09)$ \\
\hline 9 & $(1,0,0)$ & 21 & $(0,0.77,0.23)$ & 33 & $(0,0,1)$ \\
\hline 10 & $(0,0.9,0.1)$ & 22 & $(1,0,0)$ & 34 & $(0.39,0.61,0)$ \\
\hline 11 & $(1,0,0)$ & 23 & $(0,0,1)$ & 35 & $(1,0,0)$ \\
\hline 12 & $(0,0.22,0.78)$ & 24 & $(0,0,1)$ & & \\
\hline
\end{tabular}

From the above process, we give the following evaluation results, see Table IV. From the last two columns in Table IV, we know the ranking and the evaluation grade of teaching units.
From Table IV, we find that the rankings are in accord with their evaluation grades for most of teaching units except for the ranking 4 and the ranking 10 . For teaching unit JD, the evaluation result shows that the proportion of the indexes evaluated $C_{2}$ is larger than those of JS, WL and SX. The proportion of the indexes evaluated $C_{3}$ for $\mathrm{RJ}$ is larger than those of WY, SZ and CL. We find that the evaluation results accords with actual situations. The proposed teaching quality evaluating model is feasible and reliable.

TABLE IV. THE RESULTS OF ATTRIBUTE EVALUATION

\begin{tabular}{|c|c|c|c|c|}
\hline Teaching unit & $\left(\mu_{i 1}, \mu_{i 2}, \mu_{i 3}\right)$ & Score & Ranking & Grade \\
\hline HT & $(0.32,0.12,0.56)$ & 0.52 & 13 & $C_{3}$ \\
\hline $\mathrm{DZ}$ & $(0.30,0.09,0.61)$ & 0.52 & 15 & $C_{3}$ \\
\hline JD & $(0.46,0.23,0.31)$ & 0.63 & 4 & $C_{2}$ \\
\hline CL & $(0.33,0.24,0.43)$ & 0.55 & 9 & $C_{3}$ \\
\hline NY & $(0.38,0.21,0.31)$ & 0.54 & 11 & $C_{2}$ \\
\hline DQ & $(0.61,0.21,0.18)$ & 0.63 & 5 & $C_{1}$ \\
\hline $\mathrm{HX}$ & $(0.61,0.21,0.18)$ & 0.62 & 6 & $C_{1}$ \\
\hline WL & $(0.71,0.10,0.18)$ & 0.71 & 2 & $C_{1}$ \\
\hline SX & $(0.53,0.14,0.34)$ & 0.64 & 3 & $C_{1}$ \\
\hline SM & $(0.32,0.13,0.54)$ & 0.53 & 12 & $C_{3}$ \\
\hline GL & $(0.25,0.24,0.51)$ & 0.50 & 16 & $C_{3}$ \\
\hline RW & $(0.20,0.27,0.52)$ & 0.44 & 18 & $C_{3}$ \\
\hline $\mathrm{TM}$ & $(0.40,0.09,0.51)$ & 0.52 & 14 & $C_{3}$ \\
\hline $\mathrm{SZ}$ & $(0.47,0.25,0.29)$ & 0.61 & 8 & $C_{1}$ \\
\hline $\mathrm{JZ}$ & $(0.32,0.16,0.52)$ & 0.48 & 17 & $C_{3}$ \\
\hline JT & $(0.18,0.25,0.57)$ & 0.42 & 19 & $C_{3}$ \\
\hline JS & $(0.60,0.17,0.23)$ & 0.71 & 1 & $C_{1}$ \\
\hline $\mathrm{RJ}$ & $(0.36,0.12,0.52)$ & 0.55 & 10 & $C_{3}$ \\
\hline WY & $(0.44,0.15,0.41)$ & 0.62 & 7 & $C_{2}$ \\
\hline
\end{tabular}

\section{REFERENCES}

[1] P. Yang, "The Application of AHP Method on the Teaching Activities in Classroom," Math. Practice Theory, vol. 34(2), pp.32-34, 2004.

[2] L. J. Wang, "Using the Time Efficiency Analysis Method to Appraise Teaching Efficiency," Math. Stat. Manag., vol. 22(2), pp. 11-14, 2003. 
[3] L. Z.Wu, B. T. Hang, "Evaluation of Teaching Quality with Judgment of Fussy and Overall Confidence Level," J. Beijing Inst. Tech., vol. 5(2), pp. 8-9, 2003.

[4] X. H. Wang, F. H. Huang, "The Model of Teaching Quality Evaluation Based on BP Neural Networks and Its Application," High Egn. Educ. Res., vol. (5), pp. 78-81, 2007.

[5] Q. S. Cheng, "Attribute Sets and Attribute Synthetic Assessment System," Systems Engineering -Theory \&Practice, vol. 17(9), pp. 1-8, 1997.
[6] Y. L. Lu, The Mathematical Model on the System of Teachingl Quality Evaluation and Application, master thesis, Harbin Institute of Technology, 2006. 